\title{
Equatorial Guinea, a multidrug-resistant tuberculosis hotspot in Central Africa
}

\author{
To the Editor:
}

The national tuberculosis programme (NTP) of Cameroon has recently reported that an alarming number of tuberculosis (TB) patients are border-crossing from neighboring Equatorial Guinea to seek multi-drugresistant tuberculosis (MDR-TB) treatment $[1,2]$. The World Health Organization (WHO) African Region is reported to bear a low MDR-TB proportion of new tuberculosis cases $(2.1 \%)$ compared with other WHO regions [3]. Nonetheless, there are big differences between African countries, and data on MDR-TB burden are still lacking from almost half of them. Some could hide true MDR-TB hotspots, brought about by weak diagnostic and control capacities and fueled by health and social factors of vulnerability $[4,5]$.

Equatorial Guinea is a small country in Central Africa. It is made up of an insular region (capital Malabo) and an inland region (capital Bata). Its population (1.2 million) has experienced deep lying sociodemographic changes since it became a major oil producer in the early 1990s [6]. Despite the country's booming economy (its gross domestic product ranks 43rd in the world) social and health [7] indicators remain very low, ranking 138th in the Human Development Index. HIV prevalence is rising, with the last national estimation being $6.2 \%$ in $2011[8,9]$. Tuberculosis incidence rates are increasing $(172$ cases per 100000 population in 2015) and national tuberculosis treatment success rate is declining (62\% in 2014) [10]. The only tuberculosis drug resistance (DR-TB) study was conducted some fifteen years ago and indicated MDR-TB in $2.2 \%$ of new and $25 \%$ of previously treated tuberculosis cases $[11,12]$.

In the present study, we evaluated the prevalence of rifampicin-resistant tuberculosis among consecutive smear-positive tuberculosis cases notified in the two main tuberculosis units of the programme. From February 1 to April 30, 2015, a total of 310 tuberculosis cases were notified in Equatorial Guinea, of which 228 were smear-positive pulmonary tuberculosis. Bata and Malabo tuberculosis units notified 205 (89.9\%) out of the 228 smear-positive cases, all of whom were screened for enrollment. Inclusion criteria were giving verbal consent, producing a smear-positive respiratory sample and having a questionnaire filled by the attending clinician. Samples could not be collected in 26 (13\%) out of the 205 notifications, mainly because of laboratory staffing shortages. The final study sample was 179 cases ( $87 \%$ of smear-positive case notifications at these units) (table 1). The median (interquartile range) age was 30.5 years (22-40), 56\% were male, and $45.9 \%$ were HIV positive, other characteristics retrieved were place of residence/origin, occupation, previous history of tuberculosis and contact with another tuberculosis case and its outcome. The smear-positive respiratory samples of the 179 cases were collected at admission to the programme, inactivated with GeneXpert MTB/RIF sample reagent (Xpert; Cepheid, Sunnyvale, CA, USA) stored and sent at 3-week intervals to the microbiology department of Gregorio Marañon University Hospital (Madrid, Spain) where the GeneXpert test was performed. Of the 179 cases, 151 (84\%) belonged to new tuberculosis cases and $28(16 \%)$ to previously treated cases. Treatment outcomes among those previously treated were: 14 relapse, 12 treatment failure and two lost to follow-up (table 1).

Xpert MTB/RIF failed to detect DNA of Mycobacterium tuberculosis complex (MTC) in 34 (19\%) out of the 179 cases. This could be mainly related to deterioration of genetic material prior to transportation, since sample storage time was very long. The proportion of samples with MTC undetected did not differ significantly among the batches of transported samples nor according to age, smear grading, HIV, origin, treatment category (new or previously treated) or presence/absence of a risk contact. Of the 145 cases with positive detection of MTC, GeneXpert software labeled two cases as "MTC detected, rifampicin resistance

@ERSpublications

A survey of rifampicin-resistant tuberculosis in Equatorial Guinea unveiled a hot spot of MDR-TB in central Africa http://ow.ly/YeOl304cqxT

Cite this article as: Izco S, Eyene J, Pérez-Lago L, et al. Equatorial Guinea, a multidrug-resistant tuberculosis hotspot in Central Africa. Eur Respir J 2017; 49: 1600952 [https://doi.org/10.1183/ 13993003.00952-2016]. 
TABLE 1 GeneXpert results by category for tuberculosis cases enrolled in the programme during the study period and cases recruited in the survey (Bata and Malabo units)

\begin{tabular}{|c|c|c|c|c|}
\hline & Nationwide & Bata unit & Malabo unit & $\begin{array}{l}\text { Bata and Malabo } \\
\text { units combined (survey) }\end{array}$ \\
\hline Cases enrolled during the study period & 310 & $163(52)$ & $80(26)$ & $243(78)$ \\
\hline Total & 228 & 142 & 63 & $205(90)$ \\
\hline New & 196 & 131 & 53 & $184(94)$ \\
\hline Total & NA & $128(90)$ & $51(81)$ & $179(87)$ \\
\hline New & NA & $110(84)$ & $41(77)$ & $151(82)$ \\
\hline Previously treated & NA & $18(100)$ & $10(100)$ & $28(100)$ \\
\hline \multicolumn{5}{|c|}{$\begin{array}{l}\text { Cases Xpert tested with Mycobacterium tuberculosis complex } \\
\text { detection }\end{array}$} \\
\hline Total & NA & 105 (82) & $38(74)$ & $143(80)$ \\
\hline Lost to follow-up & & 1 & 1 & $2(10)$ \\
\hline \multicolumn{5}{|l|}{ Rifampicin resistance results } \\
\hline \multicolumn{5}{|l|}{ New cases $(n=123)$} \\
\hline Rifampicin susceptible & NA & 82 & 28 & 110 \\
\hline Rifampicin resistant & NA & 10 & 3 & 13 \\
\hline Rifampicin resistant \% & NA & 10.86 & 9.67 & 10.56 \\
\hline \multicolumn{5}{|l|}{ Previously treated $(n=20)$} \\
\hline Rifampicin susceptible & NA & 3 & 5 & 8 \\
\hline Relapse & & 2 & 4 & 6 \\
\hline Failure & & 0 & 0 & 0 \\
\hline Lost to follow-up & & 1 & 1 & 2 \\
\hline Rifampicin resistant & NA & 10 & 2 & 12 \\
\hline Relapse & & 3 & 0 & 3 \\
\hline
\end{tabular}

Data are presented as $\mathrm{n}$ or $\mathrm{n}(\%)$, unless otherwise stated. NA: Not applicable. Bold highlights main results.

indeterminate" which were excluded from the study. Of the remaining 143 cases (final sample size), 123 were new cases and 20 were previously treated cases.

GeneXpert gave the result of "rifampicin resistant" in $13(10.6 \%)$ out of the 123 new cases and in 12 $(60 \%)$ of the 20 previously treated cases. The latter were three of nine relapses, nine of nine failures, and zero of two lost to follow up (table 1).

Due to the lack of a drug resistant TB (DR-TB) diagnostic and treatment programme in Equatorial Guinea, all study patients with rifampicin resistance (and also those with a high risk of DR-TB and a negative or inconclusive GeneXpert results) were transferred to Cameroon as per agreement with their NTP. Of note, in 11 of the 12 previously treated resistant cases, purified DNA was sent to Madrid from Cameroon's Reference Laboratory and tested for first- and second-line resistance mutations (Anyplex II MTB/MDR; Seegene Inc., Seoul, Korea). All cases (11 out of 11) harbored rifampicin and isoniazid mutations and two (18\%) out of 11 also had gyrA mutations, which are associated with fluoroquinolone resistance. Both of these cases were previous failures of two first-line tuberculosis regimens [13]. Ciprofloxacin (and levofloxacin, to a lesser extent) is widely used for empiric treatment of febrile episodes in Equatorial Guinea.

Regarding spatial distribution, five (13\%) rifampicin resistant cases were found among the 38 cases assessed in Malabo tuberculosis unit and 20 (19\%) rifampicin resistant cases were found among the 105 cases assessed in the Bata tuberculosis unit. This unit is not only responsible for its district population but it is also increasingly loaded with patients coming from other inland districts where NTP ceased to operate. Figure 1 shows the distribution of cases based on self-reported district of origin. A multivariate analysis (Stata/IC 13.1, StataCorp, College Station, TX, USA) revealed two independent predictive factors 


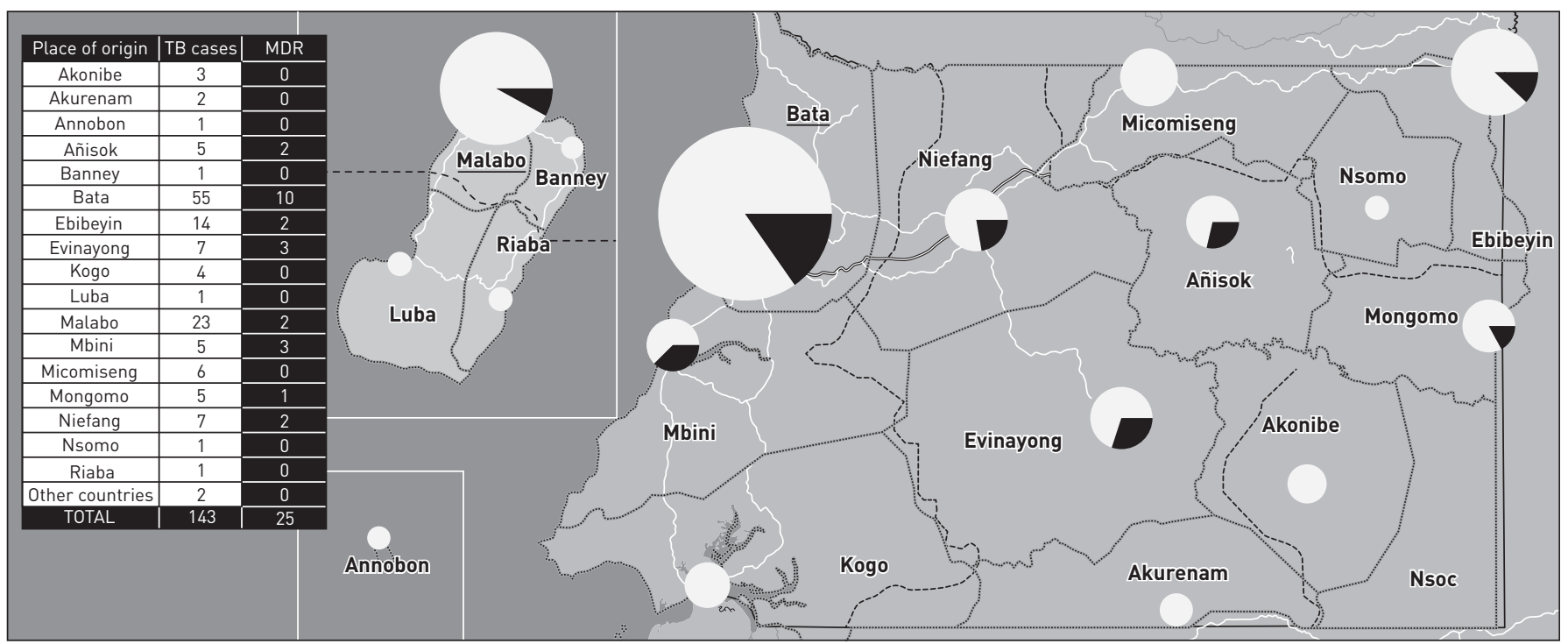

FIGURE 1 Equatorial Guinea. Districts of origin of rifampicin-resistant (black) and rifampicin non-resistant (white) tuberculosis (TB) cases of the assessment. MDR: multidrug resistant.

for RR: previously treated (OR 34.94, 95\% CI 5.13-237.87; $\mathrm{p}<0.001$ ) and prior contact with another tuberculosis case that failed or died (OR, $17.2095 \%$ CI 2.38-124.27; $\mathrm{p}=0.005)$. HIV, age, sex and region of origin did not vary significantly amongst rifampicin resistant and rifampicin non-resistant cases.

To conclude, the study found a $60 \%$ prevalence of rifampicin resistance among previously treated smear-positive pulmonary tuberculosis cases and a $10.6 \%$ prevalence of rifampicin-resistance among new smear positive pulmonary tuberculosis cases admitted to units of Equatorial Guinea's tuberculosis programme. These proportions should set off an alarm, especially for the new tuberculosis cases. The prevalence found in this group is five-fold the one estimated by WHO for the African Region [3] and three-fold the one found in Equatorial Guinea in 1999-2000 [11, 12]. A combination of factors may have fueled generation and transmission of resistant strains in this country since then. Among them, a significant reduction of resources allocated to the NTP has led to substandard drug procurements, low implementation of direct observed treatment policies and a reduction in the number of facilities detecting and treating tuberculosis in the country [14].

The survey has clear limitations. First, it was conducted on tuberculosis patients reporting exclusively at NTP facilities. Unfortunately many people with tuberculosis symptoms in Equatorial Guinea are never seen by the programme and undergo irregular treatments in private clinics or at traditional healers. Moreover, the assessment only evaluates tuberculosis notifications in the two regional capitals' tuberculosis units. These two cities are currently harboring 54\% of Equitorial Guinea's population but their units notify up to $75 \%$ of the annual national cohort of patients. Second, the assessment is based solely on GeneXpert testing. This system is recommended by WHO for surveys in contexts where culture is not available, such as Equatorial Guinea [15].

Although being previously treated and being a contact of a previous case that failed were strongly associated with resistance, nearly half of the resistant cases found in the study were new tuberculosis cases and reported no contact with a presumed DR-TB case. This finding supports the NTP's plea for implementation of universal drug sensitivity testing for all tuberculosis cases in Equatorial Guinea.

Our assessment may have unveiled a hot spot of MDR-TB in Central Africa. The high proportion (10.6\%) of new tuberculosis cases that were rifampicin resistant is particularly alarming. Results of a national evaluation are still lacking, but if the resulting figure were not very different it would rank this country among the top 20 worldwide [3].

Equatorial Guinea's national tuberculosis programme requires renewed support to strengthen and expand its general tuberculosis control measures. These are core to halt the threat that the MDR-TB epidemic poses for the country and the region. In addition, an MDR-TB programme that includes universal resistance testing of tuberculosis patients and second-line treatment for those with DR-TB is urgently needed.

Santiago Izco ${ }^{1,2}$, Juan Eyene $e^{3,4}$, Laura Pérez-Lago ${ }^{1,2,5}$, Marta Herranz ${ }^{1,2,5}$, Lucía Biyé6, Jürgen Noeske ${ }^{7}$, Felipe Pérez-García ${ }^{1}$, Deograciana Omoha $^{8}$, María-Jesús Ruiz-Serrano ${ }^{1,2,5}$, Marcial Ondó ${ }^{6}$, Teodora Mbang $^{6}$, Juan-Carlos Momo $^{8}$, Maxima Angue ${ }^{6}$, Teresa-Sabina Ebee ${ }^{6}$, Darío García-de-Viedma ${ }^{1,2,5}$ and Emilio Bouza ${ }^{1,2,5,9}$ 
${ }^{1}$ Clinical Microbiology and Infectious Diseases Dept, Gregorio Marañon University Hospital, Madrid, Spain. ${ }^{2}$ Instituto de Investigación Sanitaria Gregorio Marañón, Madrid, Spain. ${ }^{3}$ National Tuberculosis Program, Malabo, Equatorial Guinea. ${ }^{4}$ Ministerio de Sanidad y Bienestar Social, Malabo, Equatorial Guinea. ${ }^{5}$ CIBER Enfermedades respiratorias, CIBERES, Madrid, Spain. ${ }^{6}$ Inland Region Tuberculosis Reference Center, Bata, Equatorial Guinea. ${ }^{7}$ Tuberculosis Centre Jamot, Yaounde, Cameroon. ${ }^{8}$ Insular Region Tuberculosis Reference Center, Malabo, Equatorial Guinea. ${ }^{9}$ Medical School, Universidad Complutense, Madrid, Spain.

Correspondence: Santiago Izco, Kingsway 252, Maseru West, Maseru, Lesotho. E-mail: santizco@yahoo.es

Received: May 112016 | Accepted after revision: Sept 022016

Support statement: The assessment was funded by two grants for International Cooperation Projects by IiSGM (Instituto de Investigación Sanitaria Gregorio Marañon, I and II-COOP-INT 2015) and a grant from Fondation Merieux (DM/CL/ cb15). Associated activities (training, biosafety and internet communication) were funded with a Mutua Madrileña cooperation prize granted to S. Izco.

Conflict of Interest: None declared.

Acknowledgements: We are grateful to Lluís Tartera for his help with the figures and to CRCE project (funded by AECID) for helping S. Izco with accommodation and some logistical aspects in Equatorial Guinea.

\section{References}

1 Noeske J, Foe JL, Kuaban C. Cameroon's MDR-TB treatment programme jeopardised by cross-border migration. Eur Respir J 2016; 47: 684-686.

2 Matteelli A, Lönnroth $\mathrm{K}$, Mosca D, et al. Cameroon's multidrug-resistant tuberculosis treatment programme jeopardised by cross-border migration. Eur Respir J 2016; 47: 686-688.

3 World Health Organization. Global tuberculosis report. WHO/HTM/TB/2015.22. Available from www.who.int/tb/ publications/global_report/en/ Date last accessed: November 22, 2016.

4 Gehre F, Ejo M, Fissette K, et al. Shifts in mycobacterial populations and emerging drug-resistance in West and Central Africa. PloS One 2014; 9: el10393.

5 Falzon D, Mirzayev F, Wares F, et al. Multidrug-resistant tuberculosis around the world: what progress has been made? Eur Respir J 2015; 45: 150-160.

6 Ministry of Planning and Development. Equatorial Guinea Social and Demographic Survey 2015. Preliminary results. Available from www.inege.gq/publicaciones/Censo2015_Folleto_R_Preliminares.pdf Date last accessed: November 22, 2016.

7 Reuter KE, Geysimonyan A, Molina G, et al. Healthcare in Equatorial Guinea, West Africa: obstacles and barriers to care. Pan Afr Med J 2014; 19: 369.

8 ICF International, Ministry of Planning, Economic Development and Public Investment (Equatorial Guinea). Equatorial Guinea Demographic and Health Survey 2011. Available from http://dhsprogram.com/what-we-do/ survey/survey-display-314.cfm Date last accessed: November 22, 2016.

9 UNAIDS. Aids info. Data sheet. Equatorial Guinea. 2015. Available from www.unaids.org/en/regionscountries/ countries/equatorialguinea/ Date last accessed: November 22, 2016.

10 World Health Organization. Tuberculosis country profiles. Equatorial Guinea. 2015. Available from www.who.int/ $\mathrm{tb} /$ country/data/profiles/en/ Date last accessed: November 22, 2016.

11 Tudo G, Gonzalez J, Obama R, et al. Study of resistance to anti-tuberculosis drugs in five districts of Equatorial Guinea: rates, risk factors, genotyping of gene mutations and molecular epidemiology. Int J Tuberc Lung Dis 2004; 8: 15-22.

12 Tudo G, Gonzalez-Martin J, Obama R, et al. Molecular epidemiology of tuberculosis in the Bata and Malabo districts of Equatorial Guinea. Int J Tuberc Lung Dis 2004; 8: 1458-1463.

13 Falzon D, Gandhi N, Migliori G, et al. Resistance to fluoroquinolones and second-line injectable drugs: impact on multidrug-resistant TB outcomes. Eur Respir J 2013; 42: 156-168.

14 Eyene J, Izco S. [Equatorial Guinea, hope and weakness of an african national tuberculosis Program, the MDR-TB challenge]. Enfermedades Emergentes 2015; 14: 2.

15 World Health Organization. Xpert MTB/RIF implementation manual: technical and operational 'how-to'; practical considerations. 2014. Available from www.who.int/tb/publications/xpert_implem_manual/en/ Date last accessed: November 22, 2016 